\title{
Sliced microRNA targets and precise loop-first processing of MIR319 hairpins revealed by analysis of the Physcomitrella patens degradome
}

\author{
CHARLES ADDO-QUAYE, ${ }^{1,2}$ JO ANN SNYDER, ${ }^{1,3}$ YONG BUM PARK, ${ }^{1,3,4}$ YONG-FANG LI, $^{5}$ \\ RAMANJULU SUNKAR, ${ }^{5}$ and MICHAEL J. AXTELL ${ }^{1,3,4}$ \\ ${ }^{1}$ Huck Institutes of the Life Sciences, Pennsylvania State University, University Park, Pennsylvania 16802, USA \\ ${ }^{2}$ Department of Computer Science and Engineering, Pennsylvania State University, University Park, Pennsylvania 16802, USA \\ ${ }^{3}$ Department of Biology, Pennsylvania State University, University Park, Pennsylvania 16802, USA \\ ${ }^{4}$ Plant Biology Graduate Program, Pennsylvania State University, University Park, Pennsylvania 16802, USA \\ ${ }^{5}$ Department of Biochemistry and Molecular Biology, Oklahoma State University, Stillwater, Oklahoma 74078, USA
}

\begin{abstract}
Expression profiling of the $5^{\prime}$ ends of uncapped mRNAs ("degradome" sequencing) can be used to empirically catalog microRNA (miRNA) targets, to probe patterns of miRNA hairpin processing, to examine mRNA decay, and to analyze accumulation of endogenous short interfering RNA (siRNA) precursors. We sequenced and analyzed the degradome of the moss Physcomitrella patens, an important model system for functional genomic analyses in plant evolution. A total of 52 target mRNAs of 27 different Physcomitrella miRNA families were identified. Many targets of both more conserved and less conserved miRNA families encoded putative regulatory proteins. Remnants of MIRNA hairpin processing also populated the degradome data and indicated an unusual "loop-first" mode of precise processing for the MIR319 gene family. Precise loop-first processing was confirmed for native Physcomitrella, rice, and Arabidopsis MIR319 hairpins, as well as an Arabidopsis artificial MIRNA (aMIRNA) based upon a MIR319 backbone. MIR319 is thus a conserved exception to the general rule of loop-last processing of MIRNA hairpins. Loop-first MIR319 processing may contribute to the high efficacy of a widely used MIR319-based strategy for aMIRNA production in plants.
\end{abstract}

Keywords: microRNA; uncapped mRNAs; plants; Physcomitrella; genomics; second-generation sequencing

\section{INTRODUCTION}

MicroRNAs (miRNAs) are potent post-transcriptional generegulatory elements in animals, plants, and some unicellular eukaryotes. miRNAs typically fulfill this regulatory role by acting as target-selecting components for Argonaute (AGO) proteins. The molecular consequences of miRNA-mediated AGO-target interactions can vary depending upon the extent (Hutvagner and Zamore 2002), geometry (FrancoZorrilla et al. 2007), and location (Gu et al. 2009) of miRNA-target complementarity, as well as the differing capabilities of the AGO proteins in question (Montgomery et al. 2008), and likely other, as yet, undiscovered factors (Voinnet 2009). Currently known miRNA-target interac-

Reprint requests to: Michael J. Axtell, Huck Institutes of the Life Sciences, Pennsylvania State University, University Park, Pennsylvania 16802, USA; e-mail: mja18@psu.edu; fax: (814) 863-1357.

Article published online ahead of print. Article and publication date are at http://www.rnajournal.org/cgi/doi/10.1261/rna.1774909. tions in plants often result in AGO-catalyzed cleavage (e.g., "slicing") of the targeted mRNA (Mallory and Bouche 2008). Detecting the downstream remnants of AGO-catalyzed slicing by RNA ligase-mediated 5 '-rapid amplification of cDNA ends (RLM-5'-RACE) (Liu and Gorovsky 1993) is thus a powerful tool for in vivo validation of predicted miRNA targets in plants (Llave et al. 2002; Kasschau et al. 2003). For many of these plant targets, slicing alone does not account for the entirety of the miRNA-mediated repression observed at the level of protein accumulation (Chen 2004; Brodersen et al. 2008; Dugas and Bartel 2008). RLM-5'-RACE-based methods nonetheless can confirm such targets because they are indeed sliced by AGO proteins in vivo. However, at least a few plant miRNA target sites function without any detectable slicing (Axtell et al. 2006; Franco-Zorrilla et al. 2007; Brodersen et al. 2008); RLM-5'-RACE-based methods are insufficient to detect such targets.

Discovery of the sliced subset of miRNA targets can be uncoupled from a priori computational target predictions 
by an RLM-5'-RACE-based methodology which we have termed "degradome" sequencing (Addo-Quaye et al. 2008; German et al. 2008; Gregory et al. 2008). The degradome refers to the population of uncapped mRNA fragments present within a specimen; deep sequencing of the $5^{\prime}$ ends of the degradome can find the downstream remnants of miRNA-mediated slicing (Addo-Quaye et al. 2008), as well as remnants of processed MIRNA precursors (German et al. 2008) and accumulation of endogenous siRNA precursor transcripts (Gregory et al. 2008). While the sliced mRNA fragments resulting from miRNA-directed AGO cleavage account for only a tiny fraction of the degradome, they can be confidently discerned by their abundance, precise alignments with known miRNAs, and randomizations (Addo-Quaye et al. 2009). In contrast to microarray-based methods to detect uncapped mRNAs (Jiao et al. 2008; Franco-Zorrilla et al. 2009), degradome sequencing allows precise determination of $5^{\prime}$ end positions. This precision aids greatly in separating AGO-catalyzed cleavage products (which always cleave the target precisely between positions 10 and 11 relative to the guiding small RNA) from the high background of non-AGO-processed uncapped mRNAs.

The moss Physcomitrella patens is amenable to reverse genetic analysis and has a completely sequenced genome (Quatrano et al. 2007). Bryophytes like Physcomitrella are thought to closely resemble the first land plants in their gametophyte-dominated life cycles, lack of lignified vascular tissue, and morphology. A handful of abundant miRNAs are invariant between basal plants like Physcomitrella and more recently derived lineages, such as Arabidopsis and Oryza (Axtell and Bowman 2008). Additionally, a great number of seemingly bryophyte-specific miRNAs have been revealed by small RNA sequencing efforts (Arazi et al. 2005; Talmor-Neiman et al. 2006a; Axtell et al. 2007; Fattash et al. 2007). We and others have computationally predicted targets for Physcomitrella miRNAs and certain siRNAs; some of these predictions have been validated by gene-specific assays (Arazi et al. 2005; Talmor-Neiman et al. 2006a,b; Axtell et al. 2007; Fattash et al. 2007). In the present study, we identified sliced miRNA targets independent of computational predictions by degradome analysis from wild-type Physcomitrella.

Liberation of most miRNA/miRNA* duplexes from canonical MIRNA precursor stem-loops requires two cycles of cleavage by endonucleases with tandem RNase III domains: One at the loop-distal position, and another at the loop-proximal position. In animals, the two processing steps are spatially separated and performed by distinct enzymes: Drosha makes the first cut at the loop-distal position in the nucleus, while Dicer makes the second cut at the loop-proximal position in the cytoplasm (Kim et al. 2009). In certain cases ("miRtrons"), the spliceosome and subsequent debranching of intron lariats substitute for the loop-distal cleavage in animals (Okamura et al. 2007; Ruby et al. 2007). In contrast, a single Arabidopsis Dicer-like enzyme (DCL1) is genetically necessary (Park et al. 2002; Reinhart et al. 2002) and biochemically sufficient (along with noncatalytic cofactors) (Dong et al. 2008) to fully liberate most miRNA/miRNA* duplexes from hairpin precursors in the nucleus. Processing intermediates indicative of "loop-last" processing have been observed in Arabidopsis (Kurihara and Watanabe 2004; Vaucheret et al. 2006), suggesting that, as in animals, the first cleavage of plant MIRNA hairpins occurs at the loop-distal position. We found that the Physcomitrella degradome contained numerous sequences corresponding to MIRNA hairpins. The positions of these apparent DCL processing remnants generally supported the loop-last hypothesis with the prominent exception of MIR319 family hairpins. We confirmed that precise processing at loop-proximal sites occurs before processing at the loop-distal sites of Physcomitrella MIR319 hairpins. We also found that both native rice and Arabidopsis MIR319 hairpins as well as a MIR319abased artificial MIRNA ( $a M I R N A)$ were precisely processed in a loop-first manner. The MIR319 family, a member of which is widely used as a backbone for amiRNA expression in plants (Schwab et al. 2006), thus appears to be a conserved exception to the general rule of loop-last MIRNA hairpin processing in plants.

\section{RESULTS AND DISCUSSION}

\section{The Physcomitrella degradome}

A library comprised of $\sim 27$-nucleotide (nt) cDNA tags deriving from the $5^{\prime}$ ends of uncapped RNAs was prepared from polyA-enriched RNA derived from 10-d-old wildtype Physcomitrella patens protonemata. Library construction was similar to our previously described method (Addo-Quaye et al. 2008) except that reverse transcription was primed with an oligo-dT tailed adapter, MmeI restriction was replaced by EcoP15I restriction (resulting in $\sim 27$-nt instead of $\sim 21$-nt inserts), and the final $5^{\prime}$ and 3 ' adapters were modified for compatibility with the Applied Biosystems SOLiD DNA sequencing system (see the schematic in Supplemental Methods). Colorspace SOLiD reads $35 \mathrm{nt}$ in length derived from the degradome sample were sorted from a co-sequenced "filler" sample, trimmed to retain colors corresponding only to the $5^{\prime}$-most $24 \mathrm{nt}$ of the cDNA insert, and translated directly to DNAspace prior to mapping (see Materials and Methods). Each 24-nt tag represented the $5^{\prime}$ end of an uncapped mRNA. A total of 14,196,093 of the trimmed degradome tags exactly matched one or more positions in the Physcomitrella nuclear genome assembly (Fig. 1A). The sampled abundance of uncapped $5^{\prime}$ ends at each position in the genome was tabulated with abundances scaled to repeat-normalized reads per million. Genome browser-compatible files showing the aligned position(s) of each of the 2,697,912 unique 24-nt tags (in .bed format, regardless of abundance) as well 
A

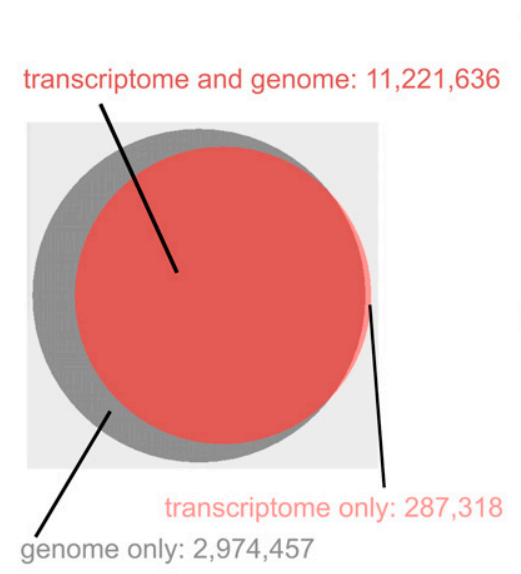

B

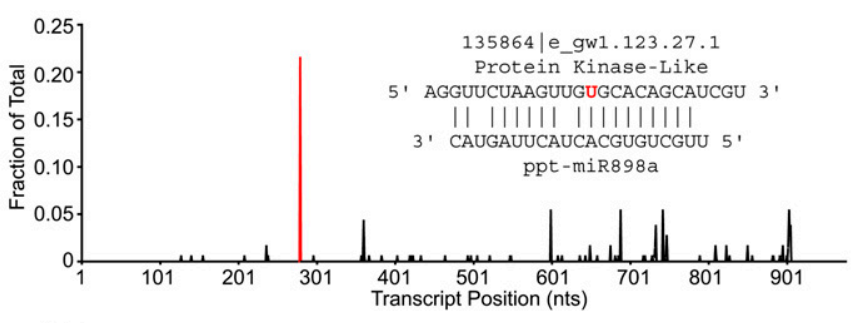

C

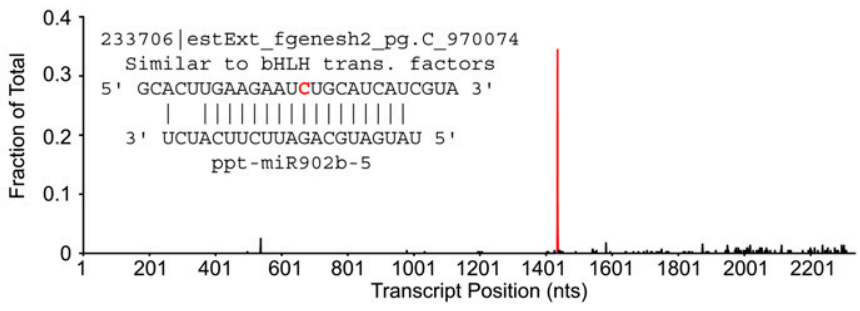

FIGURE 1. Mapping and analysis of the Physcomitrella degradome. (A) Proportional Venn diagram showing number of processed degradome tags mapping to the genome and/or the sense strand of the transcriptome. $(B, C)$ Examples of confidently identified sliced miRNA targets. The frequencies of degradome tags with $5^{\prime}$ ends at the indicated positions are shown in black, with the frequency at position 10 of the inset miRNAtarget alignment highlighted in red. Full details of all confidently identified miRNA targets are in Supplemental Table 1.

as quantitative representations of $5^{\prime}$ abundances from both the Watson and Crick genomic strands (in ".wig format") are available from our laboratory website (http://www. bio.psu.edu/people/faculty/Axtell/AxtellLab/Data.html).

Our previous predictions of Physcomitrella miRNA targets used the 35,938 mRNA annotations of the "filtered models 3" (FM3) data set (Axtell et al. 2007). We have since observed several cases in which high-confidence transcript annotations were not included in this data set but were present in a broader set of 228,057 unfiltered transcript models (UNF) produced by multiple computational and manual methods (Rensing et al. 2008). Using this expanded set of possible transcripts increased the complexity of the analysis because a single locus was almost always represented by multiple variant annotations. However, using the UNF set of variant annotations also decreased the chances of inadvertently ignoring a miRNA target. In total, 11,508,954 of the trimmed degradome reads exactly matched the sense strand of one or more of the UNF transcript models. A subset of 287,318 of these transcript-matched reads did not match the genome itself. Most of these were likely to have been tags which spanned exon-exon junctions. A subset of 2,974,457 of the genomematched reads was not matched to the sense strand of any UNF transcripts (Fig. 1A). These included tags which emanated from introns, antisense transcripts, or regions which were unannotated.

\section{Sliced miRNA targets: Comparison of predictions and empirical data}

A nonredundant set of mature Physcomitrella miRNA sequences was generated from miRBase 13.0 and used to search the degradome data for cognate slicing remnants from the UNF transcriptome using CleaveLand (AddoQuaye et al. 2009). For each potential cleavage site found, CleaveLand scored the alignment between miRNA and target using a previously described rubric (Allen et al. 2005). In addition, the quality of the degradome signal at the cleavage site was categorized based on the relative amount of degradome tags mapping to other portions of the transcript: Category one transcripts (those where the presumed cleavage site has the most degradome tags) are the highest confidence, followed by categories two and three (Addo-Quaye et al. 2008). We wished to minimize false identifications of sliced miRNA targets and therefore applied strict cutoffs to the alignment scores accepted for each category. Alignment scores of 4.5, 3.5, and 2.5 were the highest allowed for category one, two, and three targets, respectively. If one or more alignments below these initial cutoffs were found when analyzing 10 randomized miRNA controls, the cutoff score for that particular miRNA was lowered accordingly. In cases where the same cleavage site aligned to multiple miRNA queries, the miRNA family member with the best scoring alignment was retained. Filtering the CleaveLand output according to these criteria gave a total of 321 high-confidence cleavage sites from the UNF transcriptome (Supplemental Table 1). Because of the redundancy in transcript annotation, this corresponded to 52 distinct targets of 27 miRNA families (Fig. 1B,C; Table 1; Supplemental Table 1$)$. Most $(n=37)$ of the confidently identified targets were classified as category one, while a minority were category two $(n=5)$ or three $(n=10)$.

Gene-specific RLM-5'-RACE was previously used to confirm miRNA-mediated slicing of 34 protein-coding target transcripts (Talmor-Neiman et al. 2006a; Axtell et al. 2007; Fattash et al. 2007). Fifteen of these were also confidently identified in our degradome analysis (Supplemental 
TABLE 1. Sliced miRNA targets in Physcomitrella confidently identified by degradome sequencing

\begin{tabular}{|c|c|c|}
\hline $\begin{array}{l}\text { miRNA } \\
\text { family }\end{array}$ & Description of proteins encoded by target transcripts & $\begin{array}{l}\text { Number } \\
\text { of targets }\end{array}$ \\
\hline $\operatorname{miR} 156$ & SBP-box transcription factors & 3 \\
\hline miR160 & Auxin response factor & 1 \\
\hline $\operatorname{miR} 171$ & GRAS-domain transcription factors & 2 \\
\hline miR390 & Unknown & 1 \\
\hline miR408 & Plastocyanin-like & 1 \\
\hline $\operatorname{miR} 408$ & Copper-oxidase domain containing & 1 \\
\hline $\operatorname{miR} 477$ & Heat-shock protein related & 2 \\
\hline miR477 & MRP-domain ribosomal protein L29-like & 1 \\
\hline miR477 & Zinc-finger CCT-domain proteins & 2 \\
\hline miR529 & AP2-domain transcription factor & 1 \\
\hline $\operatorname{miR534}$ & Blade on Petiole 2-like BTB and ankyrin-domain proteins & 2 \\
\hline miR536 & Unknown & 1 \\
\hline $\operatorname{miR} 538$ & MADS-box, K-box containing transcription factors & 3 \\
\hline $\operatorname{miR898}$ & Protein kinase & 1 \\
\hline miR902-5p & Transcription factor-like & 4 \\
\hline miR902-5p & Unknown & 1 \\
\hline miR1023-5p & $\begin{array}{l}\text { Similar to STOP1 (sensitive to proton rhizotoxicity 1) } \\
\text { transcription factors }\end{array}$ & 2 \\
\hline $\operatorname{miR} 1027$ & Unknown & 1 \\
\hline miR1028-3p & Similar to protein arginine $\mathrm{N}$-methyltransferase & 1 \\
\hline miR1028-5p & Unknown & 1 \\
\hline miR1029 & Similar to DRE-binding transcription factor & 1 \\
\hline miR1038-5p & Histone deacetylase 2-like & 2 \\
\hline miR1039-5p & Similar to vesicle-associated membrane family proteins & 1 \\
\hline miR1043-3p & Similar to WLIM1 transcription factor & 1 \\
\hline $\operatorname{miR} 1043-3 p$ & Alcohol dehydrogenase-like & 1 \\
\hline miR1049 & PPR-repeat protein & 1 \\
\hline miR1049 & Unknown & 1 \\
\hline miR1065 & Zinc-finger domain protein similar to WIP4 transcription factor & 1 \\
\hline miR1073-5p & $\mathrm{Cu} / \mathrm{Zn}$ superoxide dismutase-like & 2 \\
\hline miR1073-5p & $\mathrm{Cu}$ oxidase domain-containing & 2 \\
\hline miR1073-5p & Glyoxal oxidase-like & 1 \\
\hline $\operatorname{miR} 1078$ & Ankyrin domain-containing protein kinase & 1 \\
\hline $\operatorname{miR} 1211$ & Molybdate transporter 1-like & 1 \\
\hline $\operatorname{miR} 1216$ & Similar to gravitropic in the light (GIL1) & 1 \\
\hline $\operatorname{miR} 1216$ & Unknown & 2 \\
\hline miR1222 & Unknown & 1 \\
\hline
\end{tabular}

Details can be found in Supplemental Tables 1-3. homology; Table 1) including $\mathrm{Cu} / \mathrm{Zn}$ oxidases (miR1073-5p), protein kinases (miR898 and miR1078), membrane transport (miR1039-5p), and ion transport (miR1211). Interestingly, for six of the 20 Physcomitrella-specific miRNAs, the target-encoded proteins were similar to DNA-binding transcription factors (Table 1; Supplemental Table 1). These six were the targets of miR538 (MADS-box factors), miR902-5p (bHLH factor), miR1023-5p (STOP1-like factor), miR1029 (DRE-binding factor), miR1043-3p (WLIM1-like factor), and miR1065 (WIP4-like zinc finger factor). Two Physcomitrella-specific miRNA families had identified targets encoding proteins similar to developmental regulators (miR534: BOP2like proteins, miR1216: GIL1-like protein), while the targets of another (miR1038-5p: Histone deacetylases) suggested a gene regulatory function. Our sampling of miRNA targets was clearly incomplete (we did not capture all previously known sliced targets, and our methodology was incapable of finding any targets regulated in the absence of slicing) and functional data are lacking. Thus, extrapolations from these data should be interpreted cautiously. It was nonetheless striking that regulation of transcripts likely involved in gene expression and/or developmental processes was a major theme for the readily identifiable sliced targets of Physcomitrella miRNAs.
Table 2). Another 12 of the confidently identified targets had been previously predicted but never confirmed (Supplemental Table 3), while the remaining seven had not previously been predicted. Degradome tags at the known cleavage sites were found for four more of the previously known targets but with category/alignment score combinations which exceeded cutoffs (Supplemental Table 2). Similarly, 14 more unconfirmed predictions also had degradome tags at the predicted cleavage sites but failed to meet our cutoffs for confidently identified targets (Supplemental Table 3).

The confidently identified targets of well-conserved miRNAs were generally homologous to those found in other plants (Table 1). The identified targets of 20 Physcomitrellaspecific miRNA families (miR534 and higher) had several different types of predicted functions (suggested by protein

\section{Remnants of MIRNA hairpin processing in degradome data}

Like AGO-catalyzed RNA cleavage, processing of primary MIRNA transcripts by plant Dicer-Like (DCL) enzymes leaves poly-adenylated remnants with a $5^{\prime}$-monophosphate. These remnants can be found by gene-specific RLM-5'RACE (Rajagopalan et al. 2006; Axtell et al. 2007). Additionally, analysis of Arabidopsis degradome data provided evidence for AGO-directed slicing of certain primary MIRNA transcripts mediated by their cognate mature miRNAs (German et al. 2008). To analyze degradome data from Physcomitrella MIRNA hairpins we first filtered the 230 MIRNA loci present in miRBase 13.0 to remove those where the annotated mature miRNA species was not sequenced in a recent sample of 367,957 small RNAs from 
wild-type 10-d-old protonemata (NCBI GEO GSM313212) (Cho et al. 2008). This had the effect of removing from consideration MIRNAs which were not highly expressed in the tissue from which the degradome sample was taken. Of the remaining 123 MIRNAs, 103 (84\%) had one or more matching degradome tags (Supplemental Tables 4, 5).

We expected that degradome tags derived from the polyadenylated remnants of DCL processing could hypothetically terminate in one of two places: Either the lower or upper cut site of the $3^{\prime}$ arm of the stem-loop (Fig. 2A, hollow blue and red triangles, respectively). The other potential source of MIRNA hairpin-derived degradome tags which we considered were AGO-catalyzed slicing remnants directed by the miRNAs themselves. The expected location of these slicing remnants is at the tenth nucleotide of complementarity to the miRNA or miRNA*, which is between the upper and lower cut sites on the arm opposite that from which the mature miRNA or miRNA* emanates (Fig. 2A, gray and white triangles). The positions expected for the remnants of the DCL-catalyzed upper cut, lower cut, and the remnant of AGO-catalyzed slicing mediated by the cognate mature miRNA were systemically estimated for each of the 103 MIRNAs using predicted secondary structures, the position of the annotated mature miRNA, and the positions and abundances of MIRNA-derived small RNAs observed from deep sequencing (Supplemental Tables 4, 5). Sites coinciding with the most abundant degradome signal on a given hairpin were scored as category one, those with a relative abundance higher than the median but lower than the maximum were scored as category two, while minor signals were categorized as category three. Remnants corresponding to the lower cut
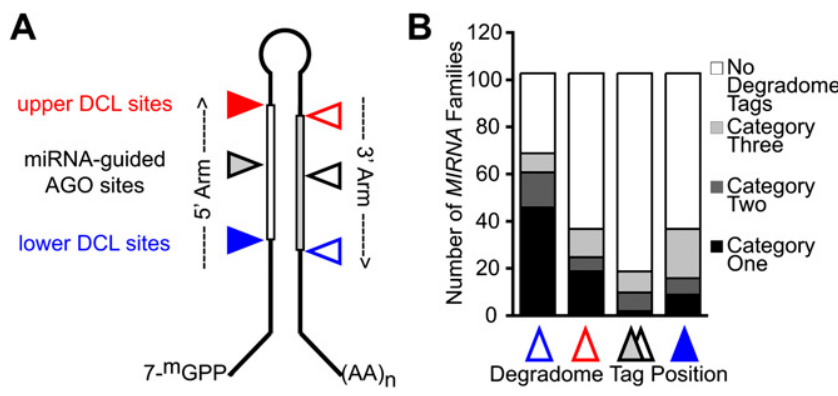

FIGURE 2. Degradome tags mapping to Physcomitrella MIRNA hairpins. (A) Schematic of a typical MIRNA hairpin showing the position of the lower DCL processing site (blue arrowheads), upper DCL processing site (red arrowheads), and the positions of potential AGO-directed cleavage by the mature miRNA or miRNA* (gray and white arrowheads). Hollow arrowheads represent expected positions of corresponding degradome tags. Degradome tags at the filled arrowheads are not due to canonical DCL processing. (B) Frequency, position, and quality of degradome tags mapping to Physcomitrella MIRNA hairpins. Category one indicates tags at that position were the most abundant relative to other positions on the hairpin, category two indicates tag abundances below the maximum but above the median, and category three indicates all others. Supporting data are in Supplemental Tables 4 and 5. site of the $3^{\prime}$ arm were found for 69 out of the 103 MIRNA hairpins; most of these (46) were category one (Fig. 2B, hollow blue triangle). Such remnants are the expected end products after DCL processing regardless of whether the upper cut had occurred second or first during miRNA biogenesis.

Unexpectedly, 37 out of the 103 hairpins showed degradome signal at the upper cut site of the $3^{\prime}$ arm (Fig. $2 \mathrm{~B}$, hollow red triangle). One interpretation of the degradome signal at this position is that precision in DCLmediated processing at the upper cut site had occurred without the lower cut having first occurred. However, contamination of the degradome sample with mature miRNAs themselves could also have resulted in degradome signals in these locations, particularly in cases where highly abundant mature miRNAs were excised from the 3' arms of the hairpin. To test if this was the case, we scored degradome signal at the positions corresponding to the lower cut site on the $5^{\prime}$ arms of the hairpins (Fig. 2A, filled blue triangle). Any degradome signal at this site could not have been due to DCL catalysis because the two tandem RNase III domains of Dicer enzymes simultaneously engage and cleave the phosphodiester backbone on both sides of the helical substrate (Zhang et al. 2004; Macrae et al. 2006). Thus, the amount of degradome signal at the position corresponding to the $5^{\prime}$ arm lower cut site served as a proxy to estimate the amount of mature miRNA contamination. The amount of degradome signal from the control $5^{\prime}$ arm lower cut sites was essentially the same as that from the $3^{\prime}$ arm upper cut sites (Fig. 2B), and both types of signals tended to correlate with instances where the mature miRNA was present on the arm of interest (Supplemental Tables 4, 5). Thus, with one important exception (see the section on conserved and precise loop-first processing of MIR319 hairpins [below]), we conclude that the degradome data offer little convincing evidence for precise DCL processing at the upper site in the absence of prior processing at the lower site. A total of 19 of the 103 hairpins had some degradome signal at positions expected for miRNA feedback slicing; however, only two of these instances were supported by strong category one signals and even in these cases the actual abundance of underlying degradome tags was very low (for an example, see miR533a in Supplemental Table 5). Thus, miRNA-mediated slicing of parental MIRNA hairpins does not seem to be widespread in Physcomitrella.

\section{Conserved and precise loop-first processing of MIR319 hairpins}

The MIR319 family is atypical in its evolutionarily consistent production of three distinct miRNA/miRNA* duplexes from unusually long hairpins (Fig. 3A; Rajagopalan et al. 2006; Talmor-Neiman et al. 2006a; Axtell et al. 2007). The mature miR319 emanates from the $3^{\prime}$ arm of the lowest 
A

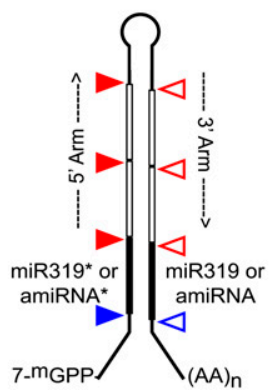

B

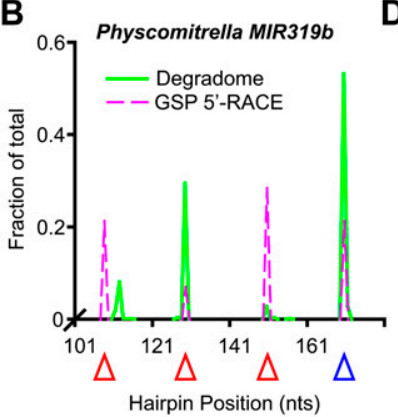

C

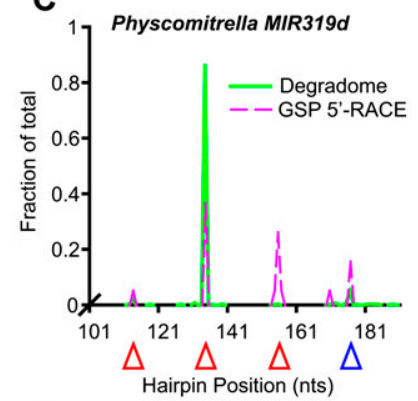

D

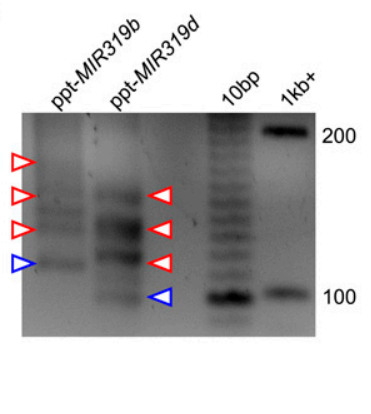

E

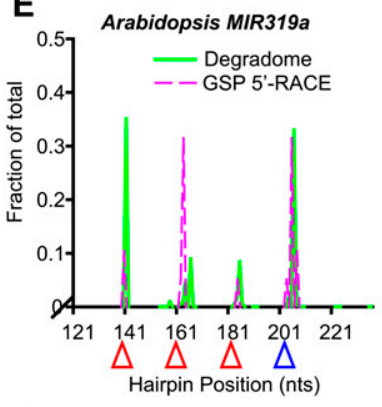

F

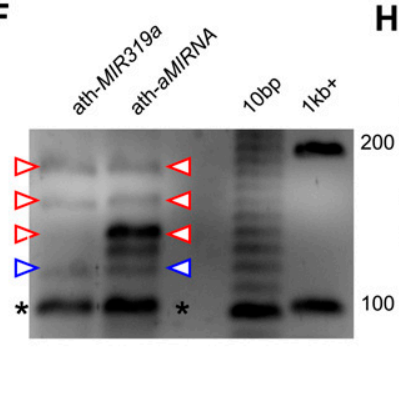

G

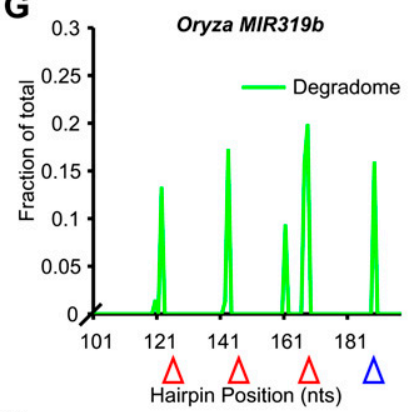

H

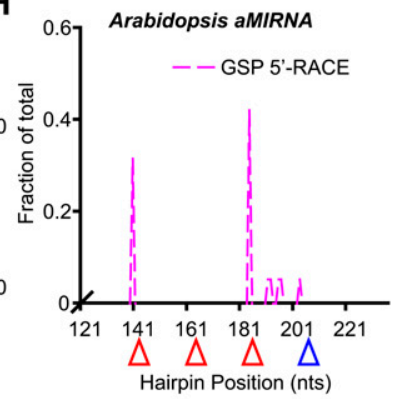

FIGURE 3. Precise and conserved "loop-first" processing of MIR319 hairpins. (A) Schematic of a MIR319 hairpin precursor. The position of mature miR319 or Arabidopsis MIR319a-based amiRNAs is indicated; the regions modified for construction of Arabidopsis MIR319a-based amiRNAs is shown in black. Arrowheads are as described in Figure 2. (B) Frequency of degradome tags (green-solid) and gene-specific (GSP) RLM-5'-RACE products (purple-dashed) mapping to the Physcomitrella MIR319b hairpin. For brevity, only the 3' arm of the hairpin is shown-degradome data on the $5^{\prime}$ arm were negligible and there were no gene-specific RLM-5'-RACE products isolated from that region. See Supplemental Tables 4 and 5 for full details. (C) As in B for Physcomitrella MIR319d. (D) Gene-specific RLM-5'-RACE products for Physcomitrella MIR319 hairpins. Arrowheads indicate the expected sizes of the corresponding processing remnants. The sizes of dsDNA molecular weight standards (10 bp and $1 \mathrm{~kb}+$ ) are indicated at right. (E) As in B for Arabidopsis MIR319a in wild-type inflorescences. See Supplemental Tables 6 and 7 for full details. (F) Gene-specific RLM-5'-RACE products for Arabidopsis MIR319a and MIR319a-based aMIRNA hairpins. Labels as in D. Asterisks indicate nonspecific artifact bands which also appeared in control reactions lacking a gene-specific primer. $(G)$ As in $B$ for Oryza MIR319b. $(H)$ As in B for Arabidopsis MIR319a-based aMIRNA over-expressing plants.

register, and is the most conserved region among MIR319 family members; however, some of the other alternative miRNAs from the middle and upper registers also show some degree of conservation (Palatnik et al. 2003; Li et al. 2005; Axtell et al. 2007). The functions of these alternative miRNAs from the middle and upper registers of MIR319 hairpins are unknown-no targets for these have been demonstrated in any species. We noticed that the members of the Physcomitrella MIR319 family showed strong degradome signals from the $3^{\prime}$ arms of all four DCL cut sites (Fig. 3B,C; Supplemental Tables 4, 5). Signals at the site separating the middle and lower register were likely to be at least partially comprised of contaminating mature miR319 (see the section on remnants of MIRNA hairpin processing in degradome data [above]), but the often stronger signals from the upper-most two cut sites did not correlate with the positions of highly abundant small RNAs (Fig. 3B,C). Importantly, independent confirmation of the existence of these remnants was obtained by gene-specific RLM-5'RACE (Fig. 3B,C). Because these gene-specific experiments isolated and sequenced long fragments (Fig. 3D), this result ruled out contamination with highly abundant mature miRNAs. Examination of previously published degradome data (Addo-Quaye et al. 2008; German et al. 2008) suggested that loop-first processing of MIR319 family hairpins also occurred in Arabidopsis (Fig. 3E; Supplemental Tables 6, 7). Loop-first processing of Arabidopsis MIR319a was confirmed by the isolation of gene-specific RLM-5'-RACE products from wild-type inflorescences (Fig. 3E,F). A degradome library constructed from Oryza sativa (rice) seedlings also contained remnants of MIR319 processing consistent with loop-first processing (Fig. 3G). We concluded that bryophyte (Physcomitrella), monocot (Oryza), and eudicot (Arabidopsis) MIR319 hairpins can be accurately processed in a "loop-first" fashion at upper cut sites without requiring an initial primary processing event at the lowest cut site.

Arabidopsis MIR319a is frequently used as a backbone for construction of aMIRNA hairpins in both Arabidopsis and Physcomitrella (Schwab et al. 2006; Khraiwesh et al. 2008). Arabidopsis MIR319a-based aMIRNAs are generated by replacing the miR319a/miR319a* duplex region of the precursor helix with a duplex engineered to produce a user-specific amiRNA; this strategy modifies the sequence, but not the predicted secondary structure, of the basal-most section of the MIR319a hairpin (Fig. 3A; Schwab et al. 
2006). Gene-specific RLM-5'-RACE using RNA prepared from aMIRNA overexpressing seedlings demonstrated that an Arabidopsis MIRNA319a-based aMIRNA was also processed in a loop-first manner in vivo (Fig. 3F,H). We concluded that the sequence of the mature miRNA or miRNA $^{*}$ does not affect loop-first processing of MIR319 family hairpins and that aMIRNAs based upon MIR319a are likely to be processed in this unexpected fashion.

\section{Conclusions}

Our sampling of sliced Physcomitrella miRNA targets contained many transcripts whose protein products had similarity to regulatory factors, including putative DNAbinding transcription factors, histone modifiers, and proteins with homology with developmentally potent Arabidopsis genes (Table 1). This result is unsurprising for miRNA families which are clearly conserved in flowering plants (Axtell and Bowman 2008), but was more unexpected for the cohort of miRNAs which to date have only been described in Physcomitrella. The confirmed targets of less-conserved miRNAs in Arabidopsis tend to be more functionally diverse than those of more-conserved miRNAs (Fahlgren et al. 2007). Our methodology was limited to discovery of miRNA targets which were sliced at detectable levels by AGO proteins, and thus we suspect we have missed many bona fide targets. Nonetheless, the miRNA families which most easily revealed their sliced targets clearly tended to be regulating the transcripts of regulatory proteins.

Precise processing of miRNA/miRNA* duplexes from precursor MIRNA hairpins is a critical step in miRNA biogenesis. In animals, the loop-distal cleavage occurs first followed by Dicer-catalyzed cleavage of the loop-proximal position. Because Dicer simply measures $\sim 22 \mathrm{nt}$ from the terminus, the first loop-distal processing event is responsible for precision. Precision is achieved either by the spliceosome (Okamura et al. 2007; Ruby et al. 2007) or by the Drosha-containing microprocessor complex (Han et al. 2006). In the latter case, the microprocesspor complex makes the first cleavage $\sim 11$ base pairs upstream of the single-stranded/double-stranded junction. This requirement of the microprocessor complex likely accounts for the very stereotypical lengths and secondary structures of most animal MIRNAs (Han et al. 2006). In contrast, the lengths and secondary structures of plant MIRNAs vary widely between precisely processed families and both cleavage events are often catalyzed by the same enzyme (DCL1 and co-factors in Arabidopsis) (Park et al. 2002; Reinhart et al. 2002; Dong et al. 2008). The cis-acting factors that promote precise processing of heterogeneously sized and structured MIRNA hairpins by a single enzyme remain largely undocumented. The loop-distal cleavage sites of Arabidopsis MIR163 (Kurihara and Watanabe 2004) and MIR168 (Vaucheret et al. 2006) are processed before the loop-proximal sites. This suggests that, like in animals, precision in plant MIRNA processing could be achieved by the selection of the first, loop-distal processing site, with the second site being selected by a simple measurement from the resulting terminus. We found that Physcomitrella MIRNA hairpins were common sources of uncapped RNAs, many of which were the likely remnants of miRNA/miRNA* excision. For most MIRNAs, our degradome data were consistent with the hypothesis of loop-last processing and the related hypothesis that precision is frequently achieved by the first, loop-distal processing event.

Our observations of uncapped MIR319 fragments which terminated at the upper positions of cleavage indicated that DCL-catalyzed processing was capable of precisely identifying the proper cleavage positions without relying upon measuring from an initial loop-distal processing event to set the register. One possible explanation for this observation is that the initiating cleavage event instead occurred at the loop proximal site, and subsequent processing events simply measured $\sim 21$ nt from the first cut, in the process liberating the three duplexes which typify the MIR319 family. A second explanation is that precision at all four of the MIR319 processing sites was achieved independent of any prior processing events. It is important to note that there is as yet no evidence that the processing remnants we have observed reflected intermediates in the production of mature miR319-it is possible that hairpins cleaved first at the upper positions were off-pathway products incapable of being completely processed to yield the miR319/miR319* duplex. The fact that loop-first processing occurred does not rule out the possibility that canonical loop-last processing is responsible for productive MIR319 maturation. However, even if loop-first processing of MIR319 hairpins was an abortive side-reaction which did not contribute to production of mature miR319, the fact remains that precise positioning of cleavage at the three upper sites consistently occurred without a pre-existing cut at the basal-most position. This suggests that unique cis-acting features dictate loop-first processing of MIR319 hairpins. Importantly, such features need not necessarily be present near the loop themselves.

Precise loop-first processing also occurred for an aMIRNA made using a widely used Arabidopsis MIR319abased strategy. It has been demonstrated that MIR319abased aMIRNA transgenes outperform MIR172a-based ones encoding identical mature amiRNAs (Schwab et al. 2006), and that an engineered variant of MIR168 produces amiRNAs of aberrant size (Vaucheret et al. 2004). One speculative explanation for these observations is that the unusual loopfirst processing of MIR319 sets the register for successive cleavages which eventually liberate the miR319/miR319* or amiRNA/amiRNA* duplex. If the cis-acting hairpin features which direct precision at the loop are indeed loop-proximal, they would be spatially separated from the loop-distal 
regions which are modified during aMIRNA construction, thus preventing modifications from affecting precision of processing. Understanding the cis-acting features which direct precise processing of both the typical loop-last plant MIRNA hairpins and of the atypical loop-first MIR319 family is an important goal for future research.

\section{MATERIALS AND METHODS}

\section{Degradome library construction}

Wild-type 10-d-old Physcomitrella patens (Gransden) protonemata were cultured, harvested, and total RNA extracted as previously described (Cho et al. 2008). Approximately $200 \mathrm{ng}$ of polyA-enriched RNA were directly ligated to a chimeric DNA/ RNA adapter containing a 3' EcoP15I site using T4 RNA Ligase 1 (New England Biolabs). After fractionation through S-400 spin columns (GE Healthcare) to deplete short RNAs, samples were reverse-transcribed with Superscript III (Invitrogen) primed with a $\mathrm{T}_{20}$ tailed adapter. After treatment with RNase H (New England Biolabs), the primary cDNA was amplified with primers specific for the $5^{\prime}$ and $3^{\prime}$ adapters and digested with EcoP15I (New England Biolabs). The entire sample was then ligated to a dsDNA adapter using T4 DNA Ligase (New England Biolabs) followed by PAGE purification of the $\sim 97-n t$ population of $5^{\prime}$ end adapter ligated fragments. After amplification, the sample was analyzed on the SOLiD 2 sequencing system (Applied Biosystems). Because the SOLiD 2 system is incapable of analyzing samples where every nucleotide is identical (the first six nucleotides of the degradome samples are the EcoP15I recognition site), our sequencing runs mixed in an irrelevant sample with SOLiD adapters from an unrelated experiment at a ratio of nine parts degradome to one part "filler." A detailed protocol including oligo sequences and a schematic flow-chart is available (Supplementary Methods). Oryza degradome sequencing on an Illumina Genome Analyzer platform using poly $(\mathrm{A})^{+}$RNA harvested from three-week-old seedlings was essentially as described by Addo-Quaye et al. (2008), and will be described more fully in another publication.

\section{Initial data analysis}

Raw colorspace reads were first parsed to separate the degradome reads from the "filler" by identifying the presence of the colors corresponding to the 6-nt EcoP15I site. Reads with the site were trimmed and retained, while reads lacking the site were discarded. Although EcoP15I cleavage is expected to chiefly generate 27-nt fragments, we have consistently observed a distribution of EcoP15I-derived cleavage sizes ranging from $\sim 25$ to $\sim 29$ nt (data not shown). By computationally trimming the $3^{\prime}$ ends to leave 24-nt reads we increased the probability that the resulting data are largely free of contamination by adapter sequences, and also removed data from the $3^{\prime}$ of the read which is typically of lower quality. The SOLiD system's two-base encoding scheme allows single color errors to cause "frameshifts" if the read is translated to DNA before mapping to a reference. Because we only wished to analyze those trimmed reads which could be exactly matched to either the Physcomitrella nuclear genome or nuclear transcriptome we directly translated into DNA space before mapping. Thus, any sequencing errors, including translation-induced frameshifts, were likely to be ignored as they would be unlikely to exactly match the reference sequences. Trimmed 24-nt DNA reads, where the first nucleotide represented an uncapped 5 ' end, were mapped to the version 1.1 Physcomitrella genome and to the unfiltered set of transcript annotations using Oligomap (Berninger et al. 2008) followed by parsing to retain only exact matches to either polarity of the genome or to the sense polarity of the transcriptome. Mapped 24-nt degradome tags have been deposited in NCBI GEO (GSE16367). Genome browser track files for the mapped data are also available from our lab website (http://www.bio.psu.edu/ people/faculty/Axtell/AxtellLab/Data.html). The Oryza degradome data have also been deposited in NCBI GEO (GSE17398).

\section{Identification of sliced miRNA targets and MIRNA processing remnants}

Sliced miRNA targets were identified using CleaveLand (AddoQuaye et al. 2009) using the unfiltered set of Physcomitrella draft genome version 1.1 transcripts and all miRBase 13.0 mature miRNA sequences as input. The initial CleaveLand output, including all matches for alignment scores up to seven, was first condensed to remove redundant cleavage sites which often resulted from multiple sequence variants from the same miRNA family. Candidate targets were further filtered to eliminate instances with alignment scores exceeding 4.5, 3.5, and 2.5 for category one, two, and three targets, respectively. Ten randomized shuffles were analyzed for each miRNA query. If any of these shuffles found a hit with an alignment score at or below the initial thresholds, the final alignment score cutoff for that query was lowered to 0.5 below the score of the random hit. Cleavage sites passing these quality filters were then collapsed to give nonredundant genes by assessing the genomic locations of overlapping transcript models (Supplemental Table 1). Previously predicted targets (Supplemental Table 3) were from Axtell et al. (2007). Degradome tags matching MIRNA hairpins were assessed by comparisons of their respective genomic coordinates (Supplemental Tables 4-7).

\section{Gene-specific RLM-5' - RACE and aMIRNA experiments}

Gene-specific RLM-5'-RACE was performed essentially as described (Axtell et al. 2007) except that the 5' RNA adapter and 3' oligo-dT primer sequences were changed for compatibility with EcoP15I degradome libraries for the SOLiD DNA sequencing system (Oligos are listed in Supplemental Methods). Arabidopsis MIR319a and aMIRNA experiments used total RNA as input instead of poly $(\mathrm{A})^{+} \mathrm{RNA}$ and were reverse-transcribed using a gene-specific oligo (5'-CCAGAACTATATATACGAAGGCAGC ATA-3'). The gene-specific oligo sequences were:

ppt-MIR319b outer: 5'-TCTTGGAACGCAGGTGCAACAGAAG ACT-3';

ppt-MIR319b inner: 5'-GGTCAGCCCAACACCACGGGAACATG AA-3';

ppt-MIR319d outer: 5'-CAGTTATCCCCTTTTATCCAGGGTTC AT-3';

ppt-MIR319d inner: 5'-TAGATCAAGAACCTTGGTACGCTCT ACA-3'; and

ath-MIR319a: 5'-GTCACTTAGTGGATCAAGCATGTTTTTGTG-3' . 
A MIR319a-based aMIRNA under the control of the 35 S promoter was designed to express an amiRNA of the sequence 5'-UUGGGC AAAAGUUAGUGGCUU- ${ }^{\prime}$ according to the WMD2 web microRNA designer (http://wmd2.weigelworld.org/cgi-bin/mirnatools. pl?page $=8$ ) using vector pRS300 as described (Schwab et al. 2006). Total RNA for RLM-5'-RACE was extracted from 10-d-old T3 homozygous aMIRNA seedlings.

\section{SUPPLEMENTAL MATERIAL}

Supplemental material can be found at http://www.rnajournal.org.

\section{ACKNOWLEDGMENTS}

We thank Craig Praul and Anton Nekrutenko for support and advice with the SOLiD DNA sequencing system, Liang Song for stimulating discussions, Sung Hyun Cho for assistance with Physcomitrella growth, and Daniel Cosgrove for facilitating production of the aMIRNA overexpressing plants. This study was supported by grant 0718051 (to M.J.A.) from the US National Science Foundation, funds from the Oklahoma Agricultural Experiment Station (to R.S.), and by Department of Energy Grant DE-FG02-84ER13179 (to Y.B.P. through this grant to Daniel Cosgrove).

Received June 12, 2009; accepted August 23, 2009.

\section{REFERENCES}

Addo-Quaye C, Eshoo TW, Bartel DP, Axtell MJ. 2008. Endogenous siRNA and miRNA targets identified by sequencing of the Arabidopsis degradome. Curr Biol 18: 758-762.

Addo-Quaye C, Miller W, Axtell MJ. 2009. CleaveLand: A pipeline for using degradome data to find cleaved small RNA targets. Bioinformatics 25: 130-131.

Allen E, Xie Z, Gustafson AM, Carrington JC. 2005. microRNAdirected phasing during trans-acting siRNA biogenesis in plants. Cell 121: 207-221.

Arazi T, Talmor-Neiman M, Stav R, Riese M, Huijser P, Baulcombe DC. 2005. Cloning and characterization of micro-RNAs from moss. Plant J 43: 837-848.

Axtell MJ, Bowman JL. 2008. Evolution of plant microRNAs and their targets. Trends Plant Sci 13: 343-349.

Axtell MJ, Jan C, Rajagopalan R, Bartel DP. 2006. A two-hit trigger for siRNA biogenesis in plants. Cell 127: 565-577.

Axtell MJ, Snyder JA, Bartel DP. 2007. Common functions for diverse small RNAs of land plants. Plant Cell 19: 1750-1769.

Berninger P, Gaidatzis D, van Nimwegen E, Zavolan M. 2008. Computational analysis of small RNA cloning data. Methods 44: 13-21.

Brodersen P, Sakvarelidze-Achard L, Bruun-Rasmussen M, Dunoyer P, Yamamoto YY, Sieburth L, Voinnet O. 2008. Widespread translational inhibition by plant miRNAs and siRNAs. Science 320: 11851190.

Chen X. 2004. A microRNA as a translational repressor of APETALA2 in Arabidopsis flower development. Science 303: 2022-2025.

Cho SH, Addo-Quaye C, Coruh C, Arif MA, Ma Z, Frank W, Axtell MJ. 2008. Physcomitrella patens DCL3 is required for 22$24 \mathrm{nt}$ siRNA accumulation, suppression of retrotransposonderived transcripts, and normal development. PLoS Genet 4: e1000314. doi: 10.1371/journal.pgen.1000314.

Dong Z, Han MH, Fedoroff N. 2008. The RNA-binding proteins HYL1 and SE promote accurate in vitro processing of pri-miRNA by DCL1. Proc Natl Acad Sci 105: 9970-9975.
Dugas DV, Bartel B. 2008. Sucrose induction of Arabidopsis miR398 represses two $\mathrm{Cu} / \mathrm{Zn}$ superoxide dismutases. Plant Mol Biol 67: 403-417.

Fahlgren N, Howell MD, Kasschau KD, Chapman EJ, Sullivan CM, Cumbie JS, Givan SA, Law TF, Grant SR, Dangl JL, et al. 2007. High-throughput sequencing of Arabidopsis microRNAs: Evidence for frequent birth and death of MIRNA genes. PLoS One 2: e219. doi: 10.1371/journal.pone.0000219.

Fattash I, Voss B, Reski R, Hess WR, Frank W. 2007. Evidence for the rapid expansion of microRNA-mediated regulation in early land plant evolution. BMC Plant Biol 7: 13. doi: 10.1186/1471-2229-7-13.

Franco-Zorrilla JM, Valli A, Todesco M, Mateos I, Puga MI, RubioSomoza I, Leyva A, Weigel D, Garcia JA, Paz-Ares J. 2007. Target mimicry provides a new mechanism for regulation of microRNA activity. Nat Genet 39: 1033-1037.

Franco-Zorrilla JM, Del Toro FJ, Godoy M, Pérez-Pérez J, LópezVidriero I, Oliveros JC, García-Casado G, Llave C, Solano R. 2009. Genome-wide identification of small RNA targets based on target enrichment and microarray hybridizations. Plant J 59: 840-850.

German MA, Pillay M, Jeong DH, Hetawal A, Luo S, Janardhanan P, Kannan V, Rymarquis LA, Nobuta K, German R, et al. 2008. Global identification of microRNA-target RNA pairs by parallel analysis of RNA ends. Nat Biotechnol 26: 941-946.

Gregory BD, O'Malley RC, Lister R, Urich MA, Tonti-Filippini J, Chen H, Millar AH, Ecker JR. 2008. A link between RNA metabolism and silencing affecting Arabidopsis development. Dev Cell 14: 854-866.

Gu S, Jin L, Zhang F, Sarnow P, Kay MA. 2009. Biological basis for restriction of microRNA targets to the $3^{\prime}$ untranslated region in mammalian mRNAs. Nat Struct Mol Biol 16: 144-150.

Han J, Lee Y, Yeom KH, Nam JW, Heo I, Rhee JK, Sohn SY, Cho Y, Zhang BT, Kim VN. 2006. Molecular basis for the recognition of primary microRNAs by the Drosha-DGCR8 complex. Cell 125: 887-901.

Hutvagner G, Zamore PD. 2002. A microRNA in a multiple-turnover RNAi enzyme complex. Science 297: 2056-2060.

Jiao Y, Riechmann JL, Meyerowitz EM. 2008. Transcriptome-wide analysis of uncapped mRNAs in Arabidopsis reveals regulation of mRNA degradation. Plant Cell 20: 2571-2585.

Kasschau KD, Xie Z, Allen E, Llave C, Chapman EJ, Krizan KA, Carrington JC. 2003. P1/HC-Pro, a viral suppressor of RNA silencing, interferes with Arabidopsis development and miRNA function. Dev Cell 4: 205-217.

Khraiwesh B, Ossowski S, Weigel D, Reski R, Frank W. 2008. Specific gene silencing by artificial microRNAs in Physcomitrella patens: An alternative to targeted gene knockouts. Plant Physiol 148: 684-693.

Kim VN, Han J, Siomi MC. 2009. Biogenesis of small RNAs in animals. Nat Rev Mol Cell Biol 10: 126-139.

Kurihara Y, Watanabe Y. 2004. Arabidopsis micro-RNA biogenesis through Dicer-like 1 protein functions. Proc Natl Acad Sci 101: 12753-12758.

Li Y, Li W, Jin YX. 2005. Computational identification of novel family members of microRNA genes in Arabidopsis thaliana and Oryza sativa. Acta Biochim Biophys Sin (Shanghai) 37: 75-87.

Liu X, Gorovsky MA. 1993. Mapping the 5' and 3' ends of Tetrahymena thermophila mRNAs using RNA ligase mediated amplification of cDNA ends (RLM-RACE). Nucleic Acids Res 21: 4954-4960.

Llave C, Xie Z, Kasschau KD, Carrington JC. 2002. Cleavage of Scarecrow-like mRNA targets directed by a class of Arabidopsis miRNA. Science 297: 2053-2056.

Macrae IJ, Zhou K, Li F, Repic A, Brooks AN, Cande WZ, Adams PD, Doudna JA. 2006. Structural basis for double-stranded RNA processing by Dicer. Science 311: 195-198.

Mallory AC, Bouche N. 2008. MicroRNA-directed regulation: To cleave or not to cleave. Trends Plant Sci 13: 359-367.

Montgomery TA, Howell MD, Cuperus JT, Li D, Hansen JE, Alexander AL, Chapman EJ, Fahlgren N, Allen E, Carrington JC. 2008. Specificity of ARGONAUTE7-miR390 interaction and dual functionality in TAS3 trans-acting siRNA formation. Cell 133: 128-141. 
Okamura K, Hagen JW, Duan H, Tyler DM, Lai EC. 2007. The mirtron pathway generates microRNA-class regulatory RNAs in Drosophila. Cell 130: 89-100.

Palatnik JF, Allen E, Wu X, Schommer C, Schwab R, Carrington JC, Weigel D. 2003. Control of leaf morphogenesis by microRNAs. Nature 425: 257-263.

Park W, Li J, Song R, Messing J, Chen X. 2002. CARPEL FACTORY, a Dicer homolog, and HEN1, a novel protein, act in microRNA metabolism in Arabidopsis thaliana. Curr Biol 12: 1484-1495.

Quatrano RS, McDaniel SF, Khandelwal A, Perroud PF, Cove DJ. 2007. Physcomitrella patens: Mosses enter the genomic age. Curr Opin Plant Biol 10: 182-189.

Rajagopalan R, Vaucheret H, Trejo J, Bartel DP. 2006. A diverse and evolutionarily fluid set of microRNAs in Arabidopsis thaliana. Genes \& Dev 20: 3407-3425.

Reinhart BJ, Weinstein EG, Rhoades MW, Bartel B, Bartel DP. 2002. MicroRNAs in plants. Genes \& Dev 16: 1616-1626.

Rensing SA, Lang D, Zimmer AD, Terry A, Salamov A, Shapiro H, Nishiyama T, Perroud PF, Lindquist EA, Kamisugi Y, et al. 2008. The Physcomitrella genome reveals evolutionary insights into the conquest of land by plants. Science 319: 64-69.

Ruby JG, Jan CH, Bartel DP. 2007. Intronic microRNA precursors that bypass Drosha processing. Nature 448: 83-86.
Schwab R, Ossowski S, Riester M, Warthmann N, Weigel D. 2006. Highly specific gene silencing by artificial microRNAs in Arabidopsis. Plant Cell 18: 1121-1133.

Talmor-Neiman M, Stav R, Frank W, Voss B, Arazi T. 2006a. Novel micro-RNAs and intermediates of micro-RNA biogenesis from moss. Plant J 47: 25-37.

Talmor-Neiman M, Stav R, Klipcan L, Buxdorf K, Baulcombe DC, Arazi T. 2006b. Identification of trans-acting siRNAs in moss and an RNA-dependent RNA polymerase required for their biogenesis. Plant J 48: 511-521.

Vaucheret H, Vazquez F, Crete P, Bartel DP. 2004. The action of ARGONAUTE1 in the miRNA pathway and its regulation by the miRNA pathway are crucial for plant development. Genes \& Dev 18: 1187-1197.

Vaucheret H, Mallory AC, Bartel DP. 2006. AGO1 homeostasis entails coexpression of MIR168 and AGO1 and preferential stabilization of miR168 by AGO1. Mol Cell 22: 129-136.

Voinnet O. 2009. Origin, biogenesis, and activity of plant microRNAs. Cell 136: 669-687.

Zhang H, Kolb FA, Jaskiewicz L, Westhof E, Filipowicz W. 2004. Single processing center models for human Dicer and bacterial RNase III. Cell 118: 57-68. 

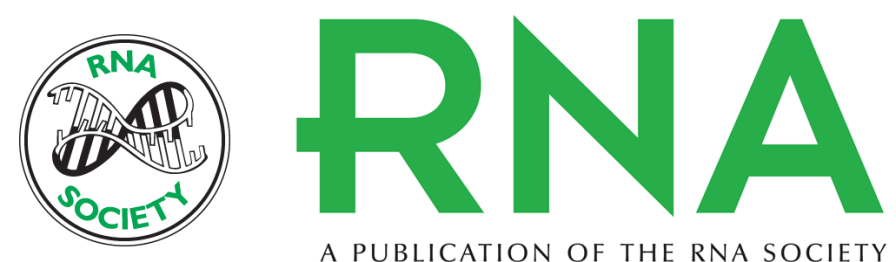

A PUBLICATION OF THE RNA SOCIETY

\section{Sliced microRNA targets and precise loop-first processing of MIR319 hairpins revealed by analysis of the Physcomitrella patens degradome}

Charles Addo-Quaye, Jo Ann Snyder, Yong Bum Park, et al.

RNA 2009 15: 2112-2121 originally published online October 22, 2009

Access the most recent version at doi:10.1261/rna.1774909

Supplemental Material

References

License

Email Alerting Service
http://rnajournal.cshlp.org/content/suppl/2009/09/22/rna.1774909.DC1

This article cites 48 articles, 15 of which can be accessed free at: http://rnajournal.cshlp.org/content/15/12/2112.full.html\#ref-list-1

Receive free email alerts when new articles cite this article - sign up in the box at the top right corner of the article or click here.

To subscribe to RNA go to:

http://rnajournal.cshlp.org/subscriptions 